\title{
LASER-AIR HYBRID ULTRASONIC TECHNIQUE FOR DYNAMIC RAILROAD INSPECTION APPLICATIONS
}

\author{
S. Kenderian ${ }^{1}$, D. Cerniglia ${ }^{2}$, B. B. Djordjevic ${ }^{3}$, G. Garcia $^{4}$ \\ ${ }^{1}$ Jet Propulsion Laboratory, Pasadena, CA $;{ }^{2}$ University of Palermo, Palermo, Italy; ${ }^{3}$ The Johns Hopkins \\ University, Baltimore, MD; ${ }^{4}$ Transportation Technology Center, Inc., Pueblo, CO.
}

\begin{abstract}
:
Laser-Air Hybrid Ultrasonic Technique (LAHUT) combines laser generation with air-coupled detection of ultrasound. The technique is non-contact and has the characteristic of operating from remote distances. Acoustic wave laser-generation apparatus can be meters away from the interrogated surface while air-coupled detection standoff can be on the order of several centimeters. The technique has the unique capability of interrogating structural materials in their true industrial environment. Dynamic tests are performed on parts with complex geometry, limited accessibility and curved surfaces. Also, dark and rough finish surfaces, which significantly reduce the efficiency of optical detection techniques, can be interrogated successfully. These characteristics make the LAHUT ideal for many industrial applications including the railroad industry. It was developed for railroad inspections targeting the most critical cracks in rails and wheels. State of the art inspection techniques available to the railroad industry often miss Vertical Split Head (VSH) and Transverse Detail Defect (TDD), which lie in unfavorable positions and orientations in the rail head. No method exists to perform dynamic inspections of the rail base or any part of the railroad wheel. Laboratory experiments were performed for the detection of TDD and Proof Of Concept (POC) field tests were performed for VSH, rail base cracks, thermal fatigue cracks along the wheel flange and tread and subsurface Shattered Rim Cracks (SRC) along the wheel tread. The results were successful and highly repeatable. The technique lends itself for digital collection and automated processing of data making the LAHUT a very strong candidate for next-generation railroad inspection technique.

\section{Introduction:}

In recent years, remote non-contact NDE techniques have been sought in an effort to improve railroad related inspection operations, more specifically, to increase the coverage of rail track and wheel testing and to perform dynamic testing of tracks and wayside inspection of wheels of a moving train. Laser, air-coupled, and water jet ultrasound transduction are the only methods that can comply with the restriction imposed by the nature of railroad operations. Water jet consumes large amounts of water and is more effective when test specimens are in an enclosure that collects and recycles water. Laser detection of ultrasound requires reflective, flat, and smooth surfaces. The dark, curved, and rough surfaces of rail tracks make optical detection of ultrasound very difficult, or impractical for industrial applications ${ }^{(1,2)}$. Air-coupled generation of ultrasound is not very effective when inspecting internal flaws in metals. More than $99 \%$ of the energy of an acoustic wave propagating from low density air to high density metals reflects back to air. Most of the remaining energy propagates mainly along the surface of the metal ${ }^{(3)}$. In the present investigation, laser generation is combined with air-coupled detection to create a noncontact and remote hybrid ultrasonic technique that can operate under industrial conditions. The proof of concept field test results, presented in this paper, show that the LAHUT is very flexible and successful in detecting a variety of cracks. Tests are performed on rail tracks detecting VSH, TDD and rail base cracks. The first two are often missed while the latter cannot be detected using conventional dynamic NDE techniques. Proof of concept tests are also performed on railroad wheels detecting thermal fatigue cracks along the wheel flange and tread and subsurface SRC along the wheel tread. No method exists for the railroad industry worldwide to perform wayside inspections of wheels on a moving train. Instead, wheels are removed and inspected in maintenance shops. Based on the proof of concept test results, two prototypes are now underway. In Italy, Tecnogamma Spa is developing a prototype for the dynamic inspection of rail tracks from a moving train while in the United States, the Transportation Technology Center (TTC), is developing a prototype for wayside dynamic inspection stations for wheels of a moving train. Experiment:

For the generation of ultrasound, an infrared Nd:YAG pulse laser is used, operating with 4-7 ns pulse width and maximum energy of $800 \mathrm{~mJ}$ per pulse. To illuminate the surface of rail tracks or wheels, the laser beam is delivered through a set of beam steering mirrors and shaped through a set of lenses. The laser source is focused to a point or a line depending on the type of flaw inspection to be performed. Water is added to the surface as a constraining 
layer to increase the amplitude of the acoustic signal. The intensity of the laser beam causes ablation. Optical observation of the surface, under 100X and 500X magnification powers, and microhardness tests show no trace of phase transformation, brittle microstructure, thermal micro cracking, or any apparent metallurgical change caused by ablation.

For the detection of ultrasound, single or multiple capacitive air-coupled transducers are used. These detectors operate with a broadband frequency spanning between $50 \mathrm{KHz}$ and $2.25 \mathrm{MHz}$. A multi-channel $250 \mathrm{KHz}$ high pass filter is used to filter out low frequency components and eliminate mechanical noise. Detected signals are received and stored in a multi-channel digital oscilloscope.

\section{Results:}

For Track inspection, the complete LAHUT and supporting assembly is mounted on a rail pushcart. Dynamic measurements are taken by pushing the assembly along the rail track at walking speeds. The tracks are tested for VSH and rail base cracks. For the wheels, two inspection stations are constructed. Each wheel set is rolled manually along the railroad track passing through one or two inspection stations, depending on the type of test performed. The detectors are positioned so that they are located above the railroad wheel, with the intent to imbed them in the tracks for the prototype. The leading edge of the wheel is detected with position sensors, which align a 2-position mirror to direct the laser beam to one of the two inspection stations and trigger the laser when the wheel is in position for the receiving transducers.

The vertical split head (VSH) is a progressive longitudinal fracture in the head of the rail, where separation along a seam spreads vertically through the head at or near the middle of the head ${ }^{(4)}$. In its advanced stages, the crack breaks the surface and a gap can be visibly seen on the running surface of the rail. Yet, these cracks are often missed by inspection techniques currently available to the railroad industry.
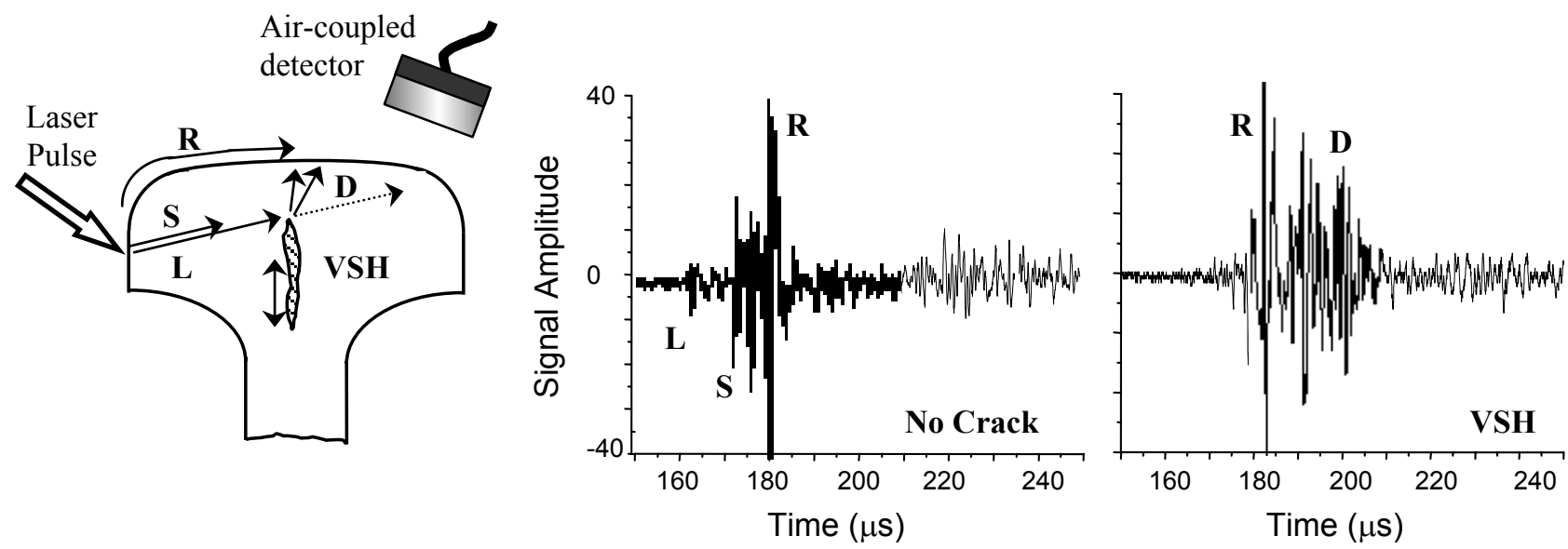

Figure 1. Test configuration showing longitudinal (L), shear (S), Rayleigh (R), and Delayed (D) waves in the rail head.

With the LAHUT, the air-coupled transducer is the closest component to the tracks, as shown in Figure 1. It is positioned $50 \mathrm{~mm}$ above the running surface of the rail. The laser source is focused to a point illuminating the field side of the railhead. In the absence of a crack, longitudinal (L) and shear (S) bulk waves propagate through the railhead, and arrive before the Rayleigh wave (R), which propagates along the surface, as shown in Fig. 1. When an internal VSH is present, the surface wave is detected with little change if any while bulk waves attenuate due to transmission. The spherical front of a point source laser-generated bulk wave interacts with the crack and results in a wide range of angular scattering from the irregular surface. Diffracted acoustic waves around the crack tip and mode-converted waves are also detected in addition to resonating waves, which radiate from the crack tip towards the surface of the railhead. Collectively, a substantial amount of a delayed signal (D), i.e., arriving after the surface wave, is received by the detector, thus indicating the presence of an internal VSH. The time-of-flight of the R wave serves as a guideline for the detection of a VSP crack. In the "No-Crack" condition, bulk modes arrive before the 
Rayleigh wave while in the "Crack" condition, the delayed signal arrives after. Using this technique, 89 measurements are collected, with 100\% success rate of finding a VSH crack.
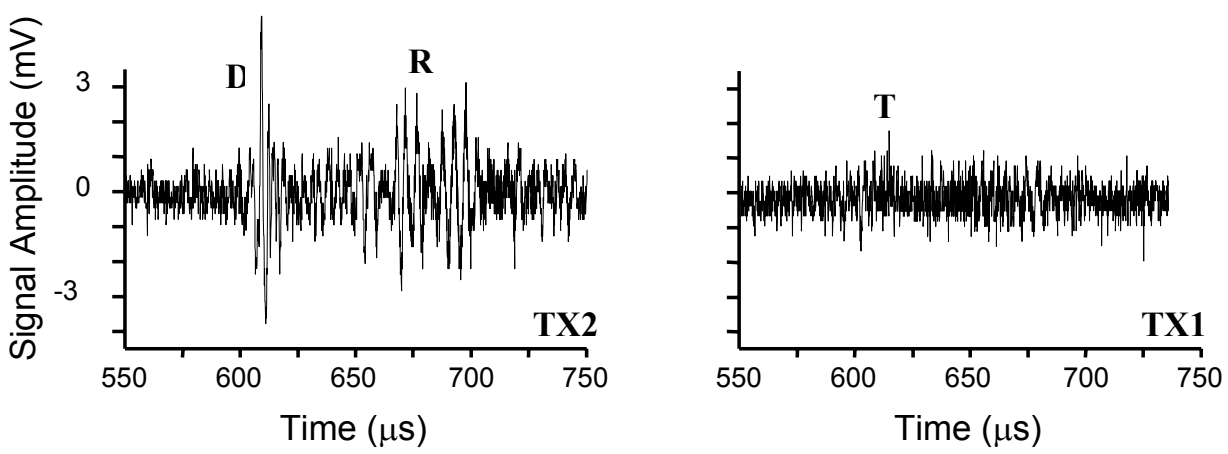

Figure 2. Acoustic signal waveforms showing direct (D), reflected (R), and transmitted (T) waves.

Rail base fractures originate from the outer edge of the base, usually caused by a nick or blow resulting in an indentation or step ${ }^{(4)}$. Initially, the crack grows relatively slowly until it has progressed approximately one-half inch into the rail. Beyond this stage, sudden rupture usually occurs resulting in complete failure. Three types of saw cuts are made to represent rail base cracks. The first type extends completely through the $13 \mathrm{~mm}$ thick base. The second and third types extend only halfway through the thickness of the base. They penetrate $6 \mathrm{~mm}$ deep from the top or bottom surfaces. In general, saw cuts are made between 13 and $19 \mathrm{~mm}$ in length measured from the edge of the rail base inward towards the center of the rail. The laser beam is focused to a $13 \mathrm{~mm}$ line on the outer edge of the rail base. Two transducers are used for detection, TX1 and TX2, positioned on opposite sides and equidistant from the laser ablated region on the rail base. When no crack is present TX1 and TX2 detect a surface acoustic wave nearly identical in strength. When a crack is present, one transducer receives an attenuated transmitted signal and the other a strong direct wave in addition to a reflected wave from the crack. Figure 2 shows the results when a $6 \mathrm{~mm}$ deep saw cut is made to the bottom surface of the $13 \mathrm{~mm}$ thick rail base. Test is performed by interrogating the top surface of the rail with a $200 \mathrm{~mm}$ air gap between the receiving transducers and the rail base and with the apparatus in motion throughout the test. The saw cut is located between the ablated region and TX1. TX1 receives an attenuated transmitted (T) signal while direct (D) and reflected (R) signals are detected with TX2. Similar analysis applies for through cracks and top surface cracks, though they are generally easier to detect. Acoustic signals are found to propagate along the rail base unaffected by clips, spikes, anchors, and other regularly spaced fasteners that are usually found in the tracks. Overall, a total of 100 measurements are taken with a $90 \%$ success rate. However, it is not possible to distinguish between the three types of cracks, i.e., through, top and bottom.
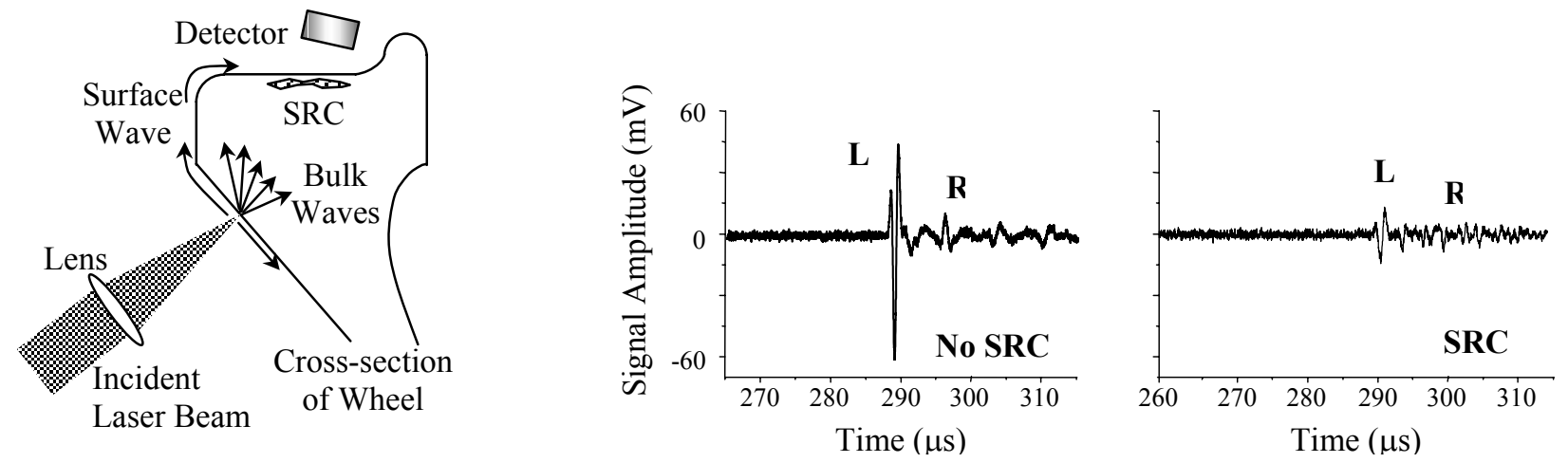

Figure 3. General setup (left) and results in the absence (middle) and presence (right) of SRC. 
The general setup and results for SRC inspection of rail wheels are shown in Figure 3. The air gap between the detector and surface of the wheel is $25 \mathrm{~mm}$ and the inclination of the detector is optimized for reception of the longitudinal bulk wave, rather than the Rayleigh surface wave, in accordance with the Snell law. This is evident from the strong Longitudinal wave component (L) and weaker Rayleigh wave component (R) detected in the "No SRC" condition in Figure 3. The presence of a subsurface SRC is detected from its interaction with the bulk waves and, depending on its depth, with the surface waves as well. Accordingly, the attenuation in the L waves is more pronounced than that of the R wave, $12 \mathrm{~dB}$ and $3 \mathrm{~dB}$ in Figure 3, respectively. The spherical front of the laser generated longitudinal wave interacts with a SRC such that detection is possible prior to the crack falling directly under the detecting transducer. The total number of measurements taken is 37 with $92 \%$ success rate. The $8 \%$ discrepancy is a result of failure to verify the presence of a SRC with a handheld flaw detector. This discrepancy may be disputed since that a flaw detector cannot receive a viable echo from a slanted or distorted surface. More details of the experiment are given in reference 5 .

Figure 4 shows the general setup for a long range surface guided wave for the inspection of fatigue cracks in the tread and flange areas of the wheel. The wheel passes through two detection stations, spaced half a revolution apart, so as to avoid the rare possibility that a crack might fall within the laser-illuminated region or directly under the detector and produce obscure effects on the received acoustic signal. Each detection assembly comprises two capacitive air-coupled transducers, as shown in Figure 4. The transducers are positioned to detect guided surface waves at $6.5^{\circ}{ }^{(2)}$ from the normal to the tangent of the curved surface. The two detectors are located $90^{\circ}$ along the wheel circumference relative to the position of the laser-illuminated region. By focusing the laser beam to a line, two surface waves propagate away from the illuminated region in a direction normal to the line. The two detectors, D1 and D2, are inclined to receive surface waves propagating in the counterclockwise (CCW) and clockwise (CW) directions, respectively, as shown in the drawing in Figure 4.
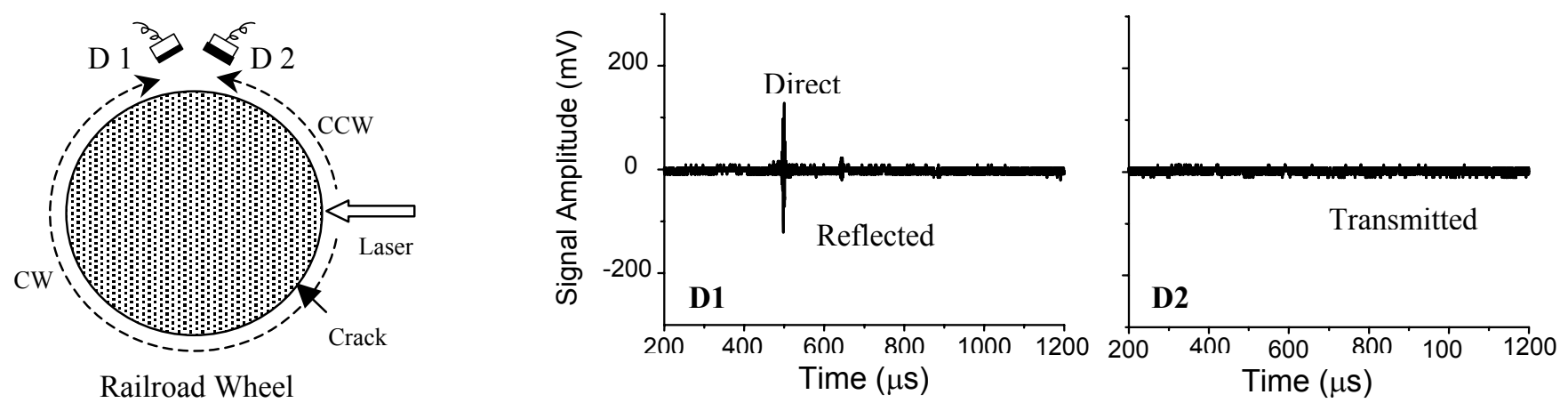

Figure 4. General setup (left) and results from D1 (middle) and D2 (right) in the presence of fatigue crack

The $1000 \mu$ s time window, in the waveforms shown in Figure 4, represents the approximate time necessary for an acoustic wave to complete one full revolution around the wheel tread circumference. A fatigue crack is present within the first quadrant in the $\mathrm{CW}$ direction from the ablated region. As a result, D1 detects a direct surface acoustic wave and a reflected wave, as shown in Figure 4, while D2 receives a highly attenuated transmitted signal. When a wheel is free of cracks, both D1 and D2 are expected to receive strong direct signals with a time of flight that corresponds with their propagation paths. The total number of measurements taken is 116 with $100 \%$ success rate.

A similar setup and test procedure applies for the inspection of the railroad wheel flange area. Three $3 \mathrm{~mm}$ deep $2.5 \mathrm{~mm}$ long saw cuts are made along the transverse plane of the flange, A, B and C. Saw cut A is made along the flange tip, $\mathrm{B}$ along the inner radius and $\mathrm{C}$ along the outer radius of the flange, Figure 5 . The laser source is focused to a point at the center of the flange tip. The generated acoustic wave propagates away from the illuminated region and insonifies the flange area. The flange behaves as a wave guide and retains a strong signal, as shown in the waveforms of Figure 6 . The $1000 \mu$ s time window, selected for the waveforms, represents the approximate time necessary for an acoustic wave to complete one full revolution around the wheel flange 
circumference. Transducers D1 and D2 are oriented to receive surface waves traveling in the CCW and CW directions, respectively, as described previously in Figure 4. A strong acoustic wave traveling one-quarter revolution of the wheel is detected via $\mathrm{D} 1$. The one-quarter revolution wave component, arriving at $498 \mu$ s as shown in Figure 6, measured $100 \mathrm{mV}$ peak to peak. It is truncated here so as to highlight the remaining components, which are indicative of the presence of a crack. Conversely, at $1060 \mu$ s, a strong acoustic wave traveling three-quarters revolutions is detected via D2. The detector D2 is oriented to receive a signal propagating in the $\mathrm{CW}$ direction. However, both detectors, D1 and D2, also receive signals propagating in the opposite directions - that is, $\mathrm{CW}$ and $\mathrm{CCW}$, respectively. The intensity of these signals is greatly reduced due to the orientation of the detectors, which are optimized for reception of waves propagating in the opposite direction.

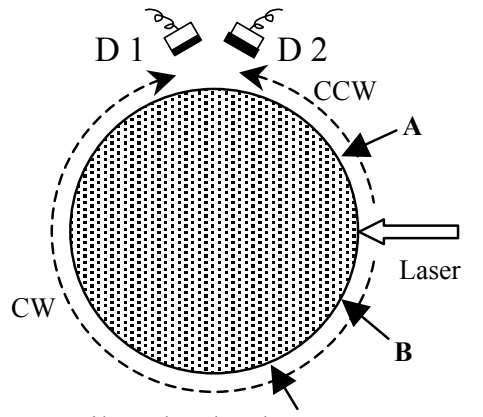

Railroad Wheel c
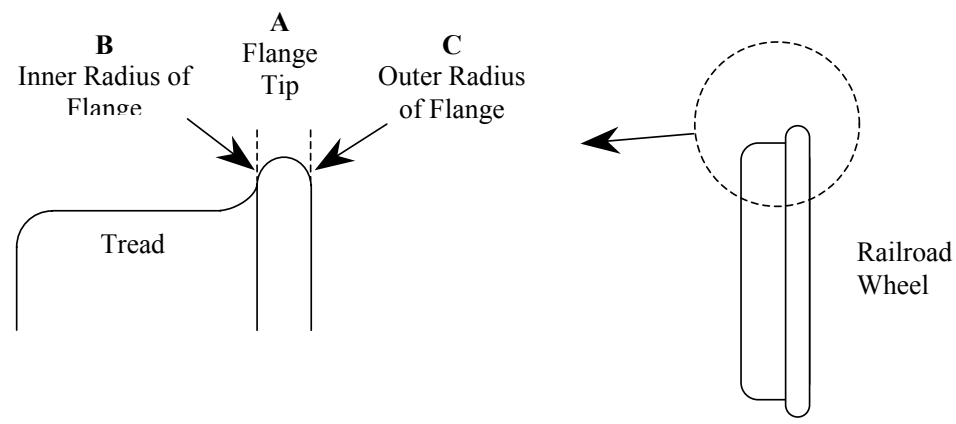

Figure 5. Schematic drawing showing the locations of the three saw cuts made on the wheel flange.
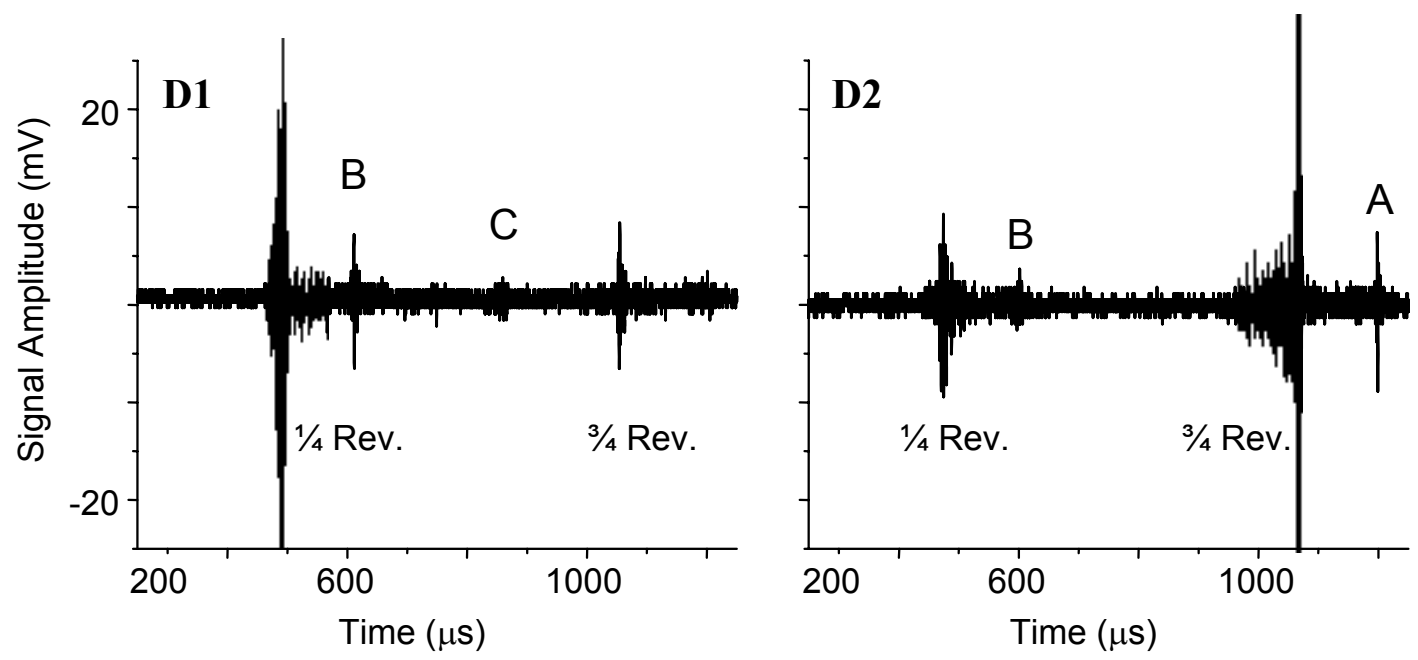

Figure 6. Acoustic waveforms received by transducer D1 (left) and D2 (right) in the presence of saw cuts $\mathrm{A}, \mathrm{B}$ and $\mathrm{C}$ along a wheel flange.

The one-quarter revolution wave component, detected by D1, is a transmitted wave through saw cut A, along the flange tip, while the three-quarters revolution wave component, detected by D2, is transmitted through saw cuts $\mathrm{B}$ and $\mathrm{C}$, along the inner and outer radii of the flange. In addition, the two detectors receive reflections from the three saw cuts, marked accordingly in Figure 6. A reflection from saw cut A propagates in the $\mathrm{CW}$ direction and is received by the second detector $\mathrm{D} 2$. The reflection is strong despite the presence of saw cuts $\mathrm{B}$ and $\mathrm{C}$ along its path. Similarly, a reflection from saw cuts B and C are received by the first detector D1. The poor detectability of 
saw cut $\mathrm{C}$ is attributed to experimental setup. Collectively, 222 measurements are taken with a average success rate of $84 \%$.

\section{Conclusions:}

A proof of concept demonstration has been performed at TTC using the Laser-Air Hybrid Ultrasonic Technique (LAHUT) for the inspection of vertical split head (VSH) and rail base cracks, at their Rail Defect Test Facility. VSH cracks frequently pass inspection while rail base cracks cannot be dynamically tested with techniques currently available to the railroad industry today. The non-contact and remote nature of the LAHUT provides it with the flexibility to perform high speed inspections of railroad tracks. In addition, the multimode and omnidirectional nature of laser-generated acoustic waves enables the technique the detection of flaws even when they are unfavorably positioned or orientated for conventional NDT techniques. The complete apparatus is mounted on a railroad pushcart. The lowest component of the apparatus is kept slightly above the running surface of the rail, thus, safe from obstacles along the tracks. Tests are performed with the pushcart in motion at walking speed. Overall, 89 measurements are collected for the VSH with a 100\% success rate. For the rail base, three types of saw cuts are made representing top surface, bottom surface and through thickness cracks. A total of 100 measurements are collected with an average success rate of $90 \%$.

The LAHUT is also successful in detecting Transverse Detail Defect (TDD) cracks in rails. Tests are performed at the Center for Nondestructive Evaluation (CNDE) at the Johns Hopkins University ${ }^{(6)}$ but are not part of the proof of concept demonstration at TTC. Due to their position on the rail track, the detection of TDD is difficult and often missed with conventional techniques. In all, VSH, rail base and TDD cracks are listed among the most critical cracks occurring in railroad tracks and can result in costly derailments ${ }^{(4,7)}$. Digital data collection makes possible for dedicated data acquisition and processing systems to automate signal interpretation in real-time to make rail inspection operations faster and more reliable. In Italy, a team of experts at Tecnogamma Spa has undertaken the task of developing a railroad track inspection prototype utilizing the LAHUT. The technique and proof of concept test results are described briefly in this paper so as to comply with the allotted space. More details on the test apparatus and results are given in reference 8 .

The proof of concept demonstration included testing railroad wheels for subsurface Shattered Rim Cracks (SRC), surface breaking thermal fatigue cracks along the tread and saw cuts along the flange. These types of cracks compromise the structural integrity of railroad wheels and can cause in-service derailments and accidents. Detection of SRC is successful to $92 \%$ accuracy. Failure to verify the presence of a SRC, using a handheld flaw detector, resulted in the disputable $8 \%$ discrepancy. Actual thermal fatigue cracks along the tread are detected to $100 \%$ accuracy but the flange test yielded less accurate results.

Three types of saw cuts are made to the flange and are labeled A, B and C, as described earlier. The three types of saw cuts are detected to $100 \%, 77 \%$ and $30 \%$ accuracy, respectively. Saw cuts B and C, along the inner and outer radii of the flange, yielded poor results in comparison with the remaining proof of concept tests. This is attributed to poor experimental setup. The selection of cylindrical lenses available to the test area and geometrical constraints imposed on the setup prohibit focusing the laser beam to a line. Ideally, for the flange test, it is preferable to focus the laser to a line extending from the inner to the outer radii of the flange, thus securing full insonofocation of the flange area. Instead, the beam is focused to a $2 \mathrm{~mm}$ point illuminating the flange tip, slightly off-center leaning towards the inner radius. As a result, better success is shown in detecting saw cuts A and B. A full understanding of the geometrical constraints and customization of the setup can improve the experiment significantly so that all parts of the inspection can be made reliable. SRCs and thermal fatigue cracks account for most railroad wheel failure incidents. Again, the technique and test results are described only briefly in this paper so as to comply with the allotted space. More details on the test apparatus and results are given in reference 5. In the United States, a multidiscipline team of engineers at TTC is utilizing the LAHUT to develop a Dynamic Detection Station (DDS) for wayside inspection of railroad wheels.

\section{References:}

1. Shant Kenderian, B. Boro Djordjevic, and Robert E. Green, Jr., "Laser based and air coupled ultrasound as noncontact nd remote techniques for testing of railroad tracks", Materials Evaluation, Vol. 60, pp. 65-70 (2002).

2. Shant Kenderian, B. Boro Djordjevic, and Robert E. Green, Jr., "Laser-Air Hybrid Ultrasonic Technique for rail wheel inspection”, Materials Evaluation, Volume 61, Number 4, pp. 505-511 (2003). 
3. Shi-Chang Wooh, "Doppler-based airborne ultrasound for detecting surface discontinuities on a moving target", Research in Nondestructive Evaluation, Vol. 12, pp. 145-166 (2001).

4. Sperry Rail Service, "Rail defect manual”, 1999.

5. Shant Kenderian, Donatella Cerniglia, B. Boro Djordjevic, Greg Garcia, Richard Morgan, Jian Sun, and Mike Snell, "Proof of concept using a laser-air of wayside railroad wheel inspection hybrid ultrasonic technique", Insight, Vol. 45, pp. 621-627 (2003).

6. Shant Kenderian, "Advanced Ultrasonic Techniques to Determine the Structural Integrity of Rail Steel", PhD Dissertation, Materials Science and Engineering Department, The Johns Hopkins University, Baltimore, Maryland, 2002.

7. Robin Clark, Surendra Singh, and Catherine Haist, "Ultrasonic characterization of defects in rails", Insight, Vol. 44, pp. 341-347 (2002).

8. Shant Kenderian, Donatella Cerniglia, B. Boro Djordjevic, Greg Garcia, Richard Morgan, Jian Sun, and Mike Snell, "Rail track field testing using laser/air hybrid ultrasonic technique", Materials Evaluation, Vol. 61 pp. 1129-1133 (2003). 